\title{
MODELAGEM DA DINÂMICA DO POTÁSSIO NO SOLO SOB IRRIGAÇÃO POR GOTEJAMENTO: VALIDAÇÃO DO MODELO
}

\author{
RENÉ N. C. RIVERA ${ }^{1}$, SERGIO N. DUARTE ${ }^{2}$, JARBAS H. DE MIRANDA ${ }^{3}$, \\ TARLEI A. BOTREL ${ }^{4}$
}

\begin{abstract}
RESUMO: A compreensão do transporte simultâneo de água e solutos a partir de uma fonte pontual permite desenvolver estratégias eficientes na fertirrigação, sendo importante no dimensionamento, operação e manejo de sistemas de irrigação localizada. Assim, o presente trabalho teve como objetivo apresentar a validação de modelo matemático desenvolvido para simular o deslocamento simultâneo de água e potássio na irrigação por gotejamento. $\mathrm{O}$ desenvolvimento do modelo baseou-se na resolução numérica de equações diferenciais parciais de segunda ordem, aplicadas à fonte puntiforme sob fluxo transiente de água e solutos. $\mathrm{O}$ experimento de validação foi conduzido no Departamento de Engenharia Rural da ESALQ/USP. O solo utilizado foi um Latossolo Vermelho, fase arenosa, série "Sertãozinho", no qual foi aplicada uma solução de cloreto de potássio com concentração de $500 \mathrm{mg} \mathrm{L}^{-1}$ de $\mathrm{K}$, com vazão de $3 \mathrm{~L} \mathrm{~h}^{-1}$, durante $2 \mathrm{~h}$. Os tempos de amostragem foram 24; 48 e $72 \mathrm{~h}$ após o início da irrigação. Observou-se que houve bom ajuste nos valores da distribuição conjunta de água e potássio no bulbo quando foram comparados os dados simulados pelo modelo e os obtidos experimentalmente. A distribuição do potássio ficou limitada às camadas mais internas do bulbo, mostrando que o deslocamento do cátion foi retardado ao interagir com a matriz do solo.
\end{abstract}

PALAVRAS-CHAVE: simulação, transporte de potássio, bulbo úmido.

\section{POTASSIUM MODELING DYNAMICS IN THE SOIL UNDER DRIP IRRIGATION: MODEL VALIDATION}

\begin{abstract}
The understanding of simultaneous water and solute transport from a punctual source allow, in fertigation, to develop efficient strategies, important in design, operation and handling of drip irrigation systems. Thus, the present work has as objective to present a mathematical model validation applied to simulate the simultaneous water and potassium dynamic under drip irrigation. The model development was based on the numeric resolution of partial differential equations of second order from point sources under transient flow of water and solute. The validation experiment was done at ESALQ/USP/Brazil. The soil used was a Red Latosol, sandy texture, "Sertãozinho" series, where a potassium chloride was applied in a $500 \mathrm{mg} \mathrm{L}^{-1}$ concentration of $\mathrm{K}$, with a $3 \mathrm{~L} \mathrm{~h}^{-1}$ flow, for $2 \mathrm{~h}$. The sampling times were $24 ; 48$ and $72 \mathrm{hr}$ after the irrigation beginning. It was observed that there was a good adjustment in the distribution of water and potassium values in the bulb when compared the data simulated by the model and experimentally obtained. The potassium distribution was limited to the most internal bulb layers, showing the ion displacement was delayed due soil matrix interactions.
\end{abstract}

KEYWORDS: simulation, potassium transport, wet bulb.

\footnotetext{
${ }^{1}$ Eng ${ }^{\circ}$ Agrônomo, Doutor em Irrigação e Drenagem, Escola Superior de Agricultura “Luiz de Queiroz" - ESALQ/USP, Professor da Universidad Mayor de San Andrés, La Paz, Bolívia, Calle Héroes del Acre, 1850, Fone: (5912) 249.1558, renechipana@yahoo.com

${ }^{2}$ Prof. Dr., Departamento de Engenharia Rural, ESALQ/USP, snduarte@esalq.usp.br

${ }^{3}$ Prof. Dr., Departamento de Ciências Exatas, ESALQ/USP, jhmirand@esalq.usp.br

${ }^{4}$ Prof. Associado, Departamento de Engenharia Rural, ESALQ/USP, tabotrel@esalq.usp.br

Recebido pelo Conselho Editorial em: 7-6-2004
}

Aprovado pelo Conselho Editorial em: 14-7-2006 


\section{INTRODUÇÃO}

Há alguns anos, a técnica da quimigação vem ganhando aceitação nos sistemas de irrigação, devido às vantagens que apresenta. Deve-se ressaltar, entretanto, que ela deve ser aplicada de modo adequado, pois, caso contrário, pode levar a problemas econômicos e a danos ao meio ambiente (KENGET et al., 1979). Segundo MMOLAWA \& OR (2000), os produtos químicos aplicados por meio da água de irrigação sofrem mudanças espacial e temporal no solo, variando sua distribuição no perfil, o que resulta em diferentes padrões de distribuição. Para CLOTHIER (1984), a compreensão do transporte simultâneo de água e solutos, em duas ou três dimensões, a partir de uma fonte pontual, permite desenvolver estratégias eficientes, sendo importante no dimensionamento, operação e manejo da fertirrigação.

Vários modelos matemáticos são usados para descrever o transporte de água e solutos em meios porosos. Modelos de infiltração para fontes puntiformes foram apresentados por BRANDT et al. (1971), WARRICK (1974) e WARRICK (1985), entre outros. Os altos custos envolvidos nas pesquisas de campo e os avanços computacionais fazem com que os modelos matemáticos, aliados às técnicas numéricas, se constituam em ferramentas bastante viáveis, possibilitando predição do destino e do comportamento da água e dos solutos na irrigação localizada. Segundo ZANINI (1991), o conhecimento da conformação dos bulbos para as diferentes condições de campo é muito importante para projetos de irrigação e para a prática da fertirrigação, e de maneira geral, os locais de maiores concentrações de $\mathrm{K}^{+}$coincidem com os locais de maiores valores de umidade, evidenciando seu caminhamento por fluxo de massa, concordando com MALAVOLTA (1980), isto é, a distribuição de potássio no solo correlacionou-se à distribuição de água no solo, indicando que se pode ter elevado controle da localização desse íon no solo em função da fertirrigação e da irrigação.

Dentre esses solutos para fornecimento do íon potássio, o cloreto de potássio é um dos fertilizantes mais empregados, devido ao fato de que os efeitos da deficiência de potássio na produtividade das plantas são bastante pronunciados.

Diante do exposto, o presente trabalho teve como objetivo apresentar a validação experimental de modelo matemático desenvolvido para simular o deslocamento simultâneo de água e potássio no solo sob irrigação por gotejamento.

\section{MATERIAL E MÉTODOS}

O desenvolvimento do modelo foi baseado na solução numérica das equações diferenciais parciais (EDP) de segunda ordem aplicadas a fontes puntiformes sob fluxo transiente, permitindo determinar a distribuição simultânea de água e de solutos no solo, em função do espaço e do tempo. A eq.(1) representa a EDP utilizada para descrever a dinâmica do íon potássio no solo.

$$
\operatorname{Fr} \frac{\partial \theta C}{\partial t}=-\frac{\partial(q C)}{\partial z}+\frac{\partial}{\partial z}\left[D \theta \frac{\partial C}{\partial z}\right]-\frac{\partial(q C)}{\partial x}+\frac{\partial}{\partial x}\left[D \theta \frac{\partial C}{\partial x}\right]
$$

em que,

Fr - fator de retardamento, adimensional;

$\theta$ - umidade atual do solo, $\mathrm{L}^{3} \mathrm{~L}^{-3}$;

D - coeficiente de dispersão-difusão, adimensional;

q - volume da solução que passa em uma unidade de área de solo na unidade de tempo, $\mathrm{L} \mathrm{T}^{-1}$, e

$\mathrm{C}$ - massa do soluto por unidade de volume de solução, $\mathrm{ML}^{-3}$.

A solução foi implementada utilizando a técnica dos volumes de controle finitos, efetuando incrementos de espaço no sentido radial e vertical, e incrementos de tempo, levando-se em consideração as hipóteses descritas por BOTREL (1988) e CRUZ (2000), sendo a região de estudo assumida como tendo forma cilíndrica e composta de anéis concêntricos (Figura 1). 


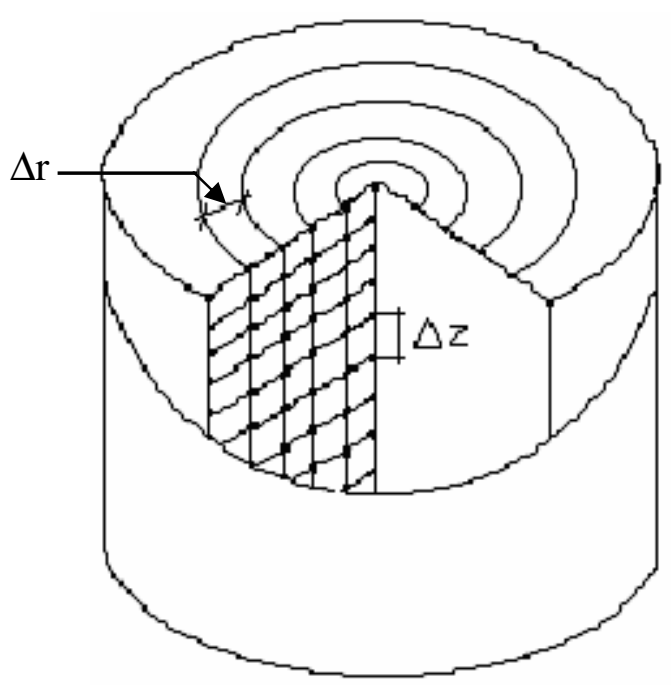

FIGURA 1. Esquema dos anéis concêntricos considerados na simulação para descrever a forma de bulbo úmido hemisférico.

O experimento de validação do modelo foi conduzido dentro de uma estufa pertencente ao Departamento de Engenharia Rural da Escola Superior de Agricultura "Luiz de Queiroz", da Universidade de São Paulo, Piracicaba - SP. O material de solo utilizado foi originado de um perfil classificado como Latossolo Vermelho, fase arenosa, série "Sertãozinho". Nas Tabelas 1; 2 e 3, são apresentadas as características químicas, conforme metodologia de RAIJ \& QUAGGIO (1983), e físico-hídricas, cuja análise granulométrica das amostras foi feita pelo método do densímetro (EMBRAPA, 1997).

TABELA 1. Características químicas da terra utilizada.

\begin{tabular}{|c|c|c|c|c|c|c|c|c|c|c|}
\hline $\mathrm{pH}$ & $\mathrm{MO}^{*}$ & $\mathrm{P}$ & $\mathrm{S}$ & $\mathrm{K}^{+}$ & $\mathrm{Ca}^{2+}$ & $\mathrm{Mg}^{2+}$ & $\mathrm{H}+\mathrm{Al}^{3+}$ & CTC & $\mathrm{V}$ & $\mathrm{m}$ \\
\hline$\left(\mathrm{CaCl}_{2}\right)$ & $\mathrm{g} \mathrm{kg}^{-1}$ & & & & ---- & $\mathrm{mol}_{\mathrm{c}} \mathrm{d}$ & -3------- & & & - \\
\hline 4,3 & 7 & 2 & 4 & 1,1 & 9 & 4 & 18 & 32,1 & 44 & 18 \\
\hline
\end{tabular}

*Matéria orgânica

TABELA 2. Características físico-hídricas da terra utilizada.

\begin{tabular}{|c|c|c|c|c|c|c|}
\hline \multicolumn{3}{|c|}{ Textura } & \multicolumn{2}{|c|}{ Densidade } & \multirow{2}{*}{ Porosidade $(\%)$} & \multirow{2}{*}{$\operatorname{Ko}\left(\mathrm{cm} \mathrm{h}^{-1}\right)$} \\
\hline Areia (\%) & Silte $(\%)$ & Argila (\%) & Global $\left(\mathrm{g} \mathrm{cm}^{-3}\right)$ & Partículas $\left(\mathrm{g} \mathrm{cm}^{-3}\right)$ & & \\
\hline 67 & 6 & 27 & 1,37 & 2,41 & 43,195 & 10,221 \\
\hline
\end{tabular}

TABELA 3. Parâmetros da curva de retenção, segundo o modelo de GENUCHTEN (1980).

\begin{tabular}{ccccc}
\hline \multicolumn{5}{c}{ Parâmetros da Curva de Retenção } \\
\hline$\theta \mathrm{r}\left(\mathrm{cm}^{3} \mathrm{~cm}^{-3}\right)$ & $\theta \mathrm{s}\left(\mathrm{cm}^{3} \mathrm{~cm}^{-3}\right)$ & $\alpha\left(\mathrm{cm}^{-1}\right)$ & $\mathrm{n}$ (adimensional) & $\mathrm{m}$ (adimensional) \\
\hline 0,113 & 0,482 & 0,029428 & 1,828069 & 0,452975 \\
\hline
\end{tabular}

Foram determinados os parâmetros de transporte de potássio no bulbo úmido a partir da elaboração de uma curva de eluição ("breakthrough curve"), sendo utilizado como soluto o cloreto de potássio, com concentração de potássio de $500 \mathrm{mg} \mathrm{L}^{-1}$. A aplicação foi feita em uma coluna de solo inserida em um tubo de PVC de $5 \mathrm{~cm}$ de diâmetro e $20 \mathrm{~cm}$ de altura. O teste estendia-se até que a concentração da solução coletada $(C)$ fosse igual à da solução aplicada $(\mathrm{Co})$. Com o intuito de auxiliar na estimativa das concentrações, foi elaborada uma curva de calibração da concentração de potássio em função da condutividade elétrica (CE) da solução. O teste foi finalizado quando a $\mathrm{CE}$ dos volumes coletados atingiram valor próximo a $1,7 \mathrm{dS} \mathrm{m}^{-1}$, o qual representava, aproximadamente, uma concentração de $500 \mathrm{mg} \mathrm{L}^{-1}$ de potássio. 
Nas amostras coletadas, determinou-se em laboratório o teor de potássio por fotometria de chama. Os volumes de solução coletados foram de $15 \mathrm{~mL}$, o que representava aproximadamente 0,082 volumes de poros. Esse volume foi controlado com balança de precisão de $0,01 \mathrm{~g}$, assumindo-se que a solução tivesse densidade igual a um. Os teores de potássio obtidos foram utilizados como dados de entrada do programa CXTFIT (desenvolvido pelo U.S. Salinity Laboratory-USDA-Riverside-CA), para determinar os valores dos parâmetros de transporte de solutos no solo: o coeficiente de dispersão-difusão (D), a velocidade da água nos poros (V), a dispersividade $(\lambda)$ e o fator de retardamento $(\mathrm{Fr})$.

Para a validação propriamente dita, o solo foi seco ao ar e posteriormente peneirado através de uma malha de $5 \mathrm{~mm}$. Em seguida, encheu-se uma caixa de polietileno, com a forma de um tronco de cone, de $2,0 \mathrm{~m}^{3}$ de capacidade, com $166 \mathrm{~cm}$ de diâmetro na parte superior, $142 \mathrm{~cm}$ de diâmetro na parte inferior e altura de $110 \mathrm{~cm}$. Posteriormente, foi aplicada ao solo uma solução de cloreto de potássio à vazão de $3 \mathrm{~L} \mathrm{~h}^{-1}$, durante $2 \mathrm{~h}$. Na coleta de amostras para a determinação da umidade do solo e da concentração de potássio, empregou-se um trado do tipo "caneco", sendo adaptado um sistema de encamisamento do orifício de coleta com um tubete de PVC, para evitar a contaminação das camadas inferiores. O diâmetro de coleta com o trado foi de $4,9 \mathrm{~cm}$, possuindo altura de $10 \mathrm{~cm}$, sendo o volume de solo coletado em cada amostragem igual a $190 \mathrm{~cm}^{3}$, o que equivalia aproximadamente a $260 \mathrm{~g}$ de solo seco. O tubete tinha diâmetro interno de 4,72 cm, sendo inferior ao diâmetro da ponta do trado, o que facilitou o encamisamento gradual, conforme se aprofundava.

Os pontos de amostragem foram localizados ao longo de uma malha formada pelos anéis concêntricos, tomando-se como eixo central o ponto em que estava localizado o emissor. Os tempos de amostragem foram: antes da irrigação, 24; 48 e 72 h após o início da irrigação. As amostras foram retiradas a dez profundidades $(0 \mathrm{a} 100 \mathrm{~cm})$ e a oito distâncias radiais $(0 \mathrm{a} 80 \mathrm{~cm})$, totalizando 90 amostras em cada tempo.

A amostragem para a determinação da concentração de potássio na solução do solo foi realizada juntamente com a amostragem de umidade. A determinação de potássio foi feita com base na retirada do extrato de saturação do solo. Primeiro preparou-se uma pasta saturada com o solo que foi retirado, deixando-o em repouso por $24 \mathrm{~h}$. Posteriormente, o extrato foi retirado por meio de bomba de vácuo para ser submetido à análise. A concentração foi corrigida em função das umidades das amostras e das pastas.

\section{RESULTADOS E DISCUSSÃO}

$\mathrm{Na}$ Tabela 4, são apresentados os parâmetros de transporte do potássio obtidos com o modelo CXTFIT. Com base nessas informações e naquelas contidas nas Tabelas 2 e 3, foram efetuadas as simulações da distribuição simultânea de água e potássio.

TABELA 4. Parâmetros de transporte do potássio obtidos com o modelo CXTFIT.

\begin{tabular}{cccc}
\hline \multicolumn{4}{c}{ Parâmetros de Transporte do Potássio } \\
\hline $\mathrm{V}\left(\mathrm{cm} \mathrm{min}^{-1}\right)$ & $\mathrm{D}\left(\mathrm{cm}^{2} \mathrm{~min}^{-1}\right)$ & $\mathrm{R}($ adimensional $)$ & $\lambda(\mathrm{cm})$ \\
\hline 1,489 & 2,705 & 4,730 & 1,816655 \\
\hline
\end{tabular}

Antes da irrigação, a umidade do solo variou, nas diferentes camadas, entre 0,119 e $0,128 \mathrm{~cm}^{3} \mathrm{~cm}^{-3}$, sendo baixa e quase constante ao longo do solo. $O$ conteúdo inicial de potássio na solução do solo também foi baixo, variando entre 16,1 e $23,4 \mathrm{mg} \mathrm{L}^{-1}$, com valor médio de $20,5 \mathrm{mg} \mathrm{L}^{-1}$.

De acordo com os dados obtidos, o conteúdo de umidade do solo dentro do bulbo após $24 \mathrm{~h}$ da irrigação, para as duas situações (dados simulados e observados), variou na faixa de 0,15 a $0,20 \mathrm{~cm}^{3} \mathrm{~cm}^{-3}$, sendo que o bulbo tinha na superfície raio de aproximadamente $30 \mathrm{~cm} \mathrm{e}$ profundidade de cerca de $40 \mathrm{~cm}$, adquirindo, portanto, forma quase hemisférica, semelhante ao 
encontrado por BOTREL (1988). Fora da região do bulbo, praticamente não houve mudança no conteúdo de água. $\mathrm{O}$ valor da umidade medida dentro do bulbo representa pouco menos da metade da umidade de saturação $\left(0,482 \mathrm{~cm}^{3} \mathrm{~cm}^{-3}\right)$, pois, no intervalo de tempo que decorreu desde a finalização da irrigação até o momento da amostragem, houve sensível redistribuição da solução, tal como citado por CRUZ (2000). Para os tempos de distribuição de 48 e 72 h após a irrigação, observou-se que as dimensões do bulbo ficaram quase constantes, para ambas as situações; entretanto, houve ligeira diminuição da umidade, principalmente nas células (espaço entre um anel e o subseqüente) próximas ao ponto do emissor, o que levou ao acréscimo da umidade das células adjacentes (redistribuição interna).

Em geral, os valores de umidade obtidos experimentalmente se apresentaram semelhantes aos simulados pelo modelo, sendo as pequenas diferenças atribuídas, possivelmente, aos erros experimentais e às limitações do modelo (BOTREL, 1988; CRUZ, 2000).

Com respeito à distribuição do potássio, nas Tabelas 5 e 6 e na Figura 2, é apresentada a concentração do íon nas diferentes células, após $24 \mathrm{~h}$ da irrigação, para as duas situações (dados medidos e simulados). Pode-se verificar que o potássio foi retido nas camadas superficiais, sendo que tanto para os valores medidos quanto para os simulados, as maiores concentrações ficaram contidas em um raio de $10 \mathrm{~cm}$ na superfície e profundidade de 0 a $20 \mathrm{~cm}$, onde as concentrações de potássio variaram de 62 até $817 \mathrm{mg} \mathrm{L}^{-1}$. O volume de solo contendo potássio em quantidades consideráveis, ou seja, maiores do que $200 \mathrm{mg} \mathrm{L}^{-1}$ ("bulbo de potássio"), foi menor que o bulbo úmido, isto é, o deslocamento do cátion não acompanhou em sua totalidade o deslocamento da água (expressado pelo fator de retardamento), provavelmente pela adsorção de potássio ao complexo de troca do solo.

Os valores da concentração de potássio simulados pelo modelo apresentaram-se semelhantes aos observados no teste, seguindo o mesmo padrão de distribuição. Os valores obtidos experimentalmente, entretanto, mostraram-se inferiores aos gerados pelo modelo, o que pode ser devido à interação do potássio com os outros cátions presentes no complexo de troca do solo, como o $\mathrm{Ca}^{2+}, \mathrm{Na}^{+}, \mathrm{Mg}^{2+}$ e o $\mathrm{Al}^{3+}$ (MMOLAWA \& OR, 2000). Para tempo de redistribuição de 48 e $72 \mathrm{~h}$ após a irrigação, verificou-se que o conteúdo de potássio nas células do bulbo permaneceu quase inalterado, embora a concentração desse elemento tenha se elevado como resultado da diminuição do teor de água nas células. A diferença entre os valores simulados e os observados esteve em aproximadamente 10\%, havendo, assim, 90\% de confiabilidade, acreditando-se que as diferenças foram devidas às razões já explicadas.

TABELA 5. Concentração de potássio na solução do solo $\left(\mathrm{mg} \mathrm{L}^{-1}\right)$, simulada pelo modelo, $24 \mathrm{~h}$ após a irrigação.

\begin{tabular}{crrrrrrrrr}
\hline $\begin{array}{c}\text { Profundidade } \\
(\mathrm{cm})\end{array}$ & \multicolumn{1}{c}{ Distância Radial $(\mathrm{cm})$} \\
\hline $0-10$ & 817,458 & \multicolumn{1}{c}{10} & 20 & 30 & 40 & 50 & 60 & 70 & 80 \\
$10-20$ & 398,168 & 62,900 & 42,811 & 19,635 & 21,060 & 23,330 & 23,400 & 23,400 & 23,400 \\
$20-30$ & 87,038 & 20,866 & 17,784 & 20,660 & 22,459 & 22,481 & 22,500 & 22,500 & 22,500 \\
$30-40$ & 22,003 & 18,123 & 20,476 & 21,809 & 21,822 & 21,822 & 21,820 & 21,820 & 21,820 \\
$40-50$ & 18,832 & 20,216 & 21,059 & 21,069 & 21,069 & 21,069 & 21,070 & 21,070 & 21,070 \\
$50-60$ & 20,289 & 20,417 & 20,419 & 20,420 & 20,420 & 20,420 & 20,420 & 20,420 & 20,420 \\
$60-70$ & 20,130 & 20,130 & 20,130 & 20,130 & 20,130 & 20,130 & 20,130 & 20,130 & 20,130 \\
$70-80$ & 20,100 & 20,100 & 20,100 & 20,100 & 20,100 & 20,100 & 20,100 & 20,100 & 20,100 \\
$80-90$ & 18,940 & 18,940 & 18,940 & 18,940 & 18,940 & 18,940 & 18,940 & 18,940 & 18,940 \\
$90-100$ & 16,070 & 16,070 & 16,070 & 16,070 & 16,070 & 16,070 & 16,070 & 16,070 & 16,070 \\
\hline
\end{tabular}


TABELA 6. Concentração de potássio na solução do solo $\left(\mathrm{mg} \mathrm{L}^{-1}\right)$, observada após $24 \mathrm{~h}$ da irrigação.

\begin{tabular}{cccccccccc}
\hline $\begin{array}{c}\text { Profundidade } \\
(\mathrm{cm})\end{array}$ & 0 & 10 & 20 & 30 & 40 & 50 & 60 & 70 & 80 \\
\hline $0-10$ & 787,215 & 194,230 & 44,503 & 31,258 & 21,543 & 17,289 & 18,497 & 23,400 & 23,400 \\
$10-20$ & 312,681 & 98,059 & 20,313 & 21,803 & 20,249 & 20,280 & 20,280 & 20,280 & 20,280 \\
$20-30$ & 98,861 & 54,287 & 18,259 & 18,258 & 22,500 & 22,500 & 22,500 & 22,500 & 22,500 \\
$30-40$ & 65,007 & 21,369 & 16,293 & 21,820 & 21,820 & 21,820 & 21,820 & 21,820 & 21,820 \\
$40-50$ & 24,915 & 13,860 & 14,368 & 21,070 & 21,070 & 21,070 & 21,070 & 21,070 & 21,070 \\
$50-60$ & 19,315 & 16,972 & 15,683 & 20,420 & 20,420 & 20,420 & 20,420 & 20,420 & 20,420 \\
$60-70$ & 18,735 & 16,759 & 16,893 & 20,130 & 20,130 & 20,130 & 20,130 & 20,130 & 20,130 \\
$70-80$ & 15,724 & 20,100 & 20,100 & 20,100 & 20,100 & 20,100 & 20,100 & 20,100 & 20,100 \\
$80-90$ & 16,252 & 18,940 & 18,940 & 18,940 & 18,940 & 18,940 & 18,940 & 18,940 & 18,940 \\
$90-100$ & 18,247 & 16,070 & 16,070 & 16,070 & 16,070 & 16,070 & 16,070 & 16,070 & 16,070 \\
\hline
\end{tabular}

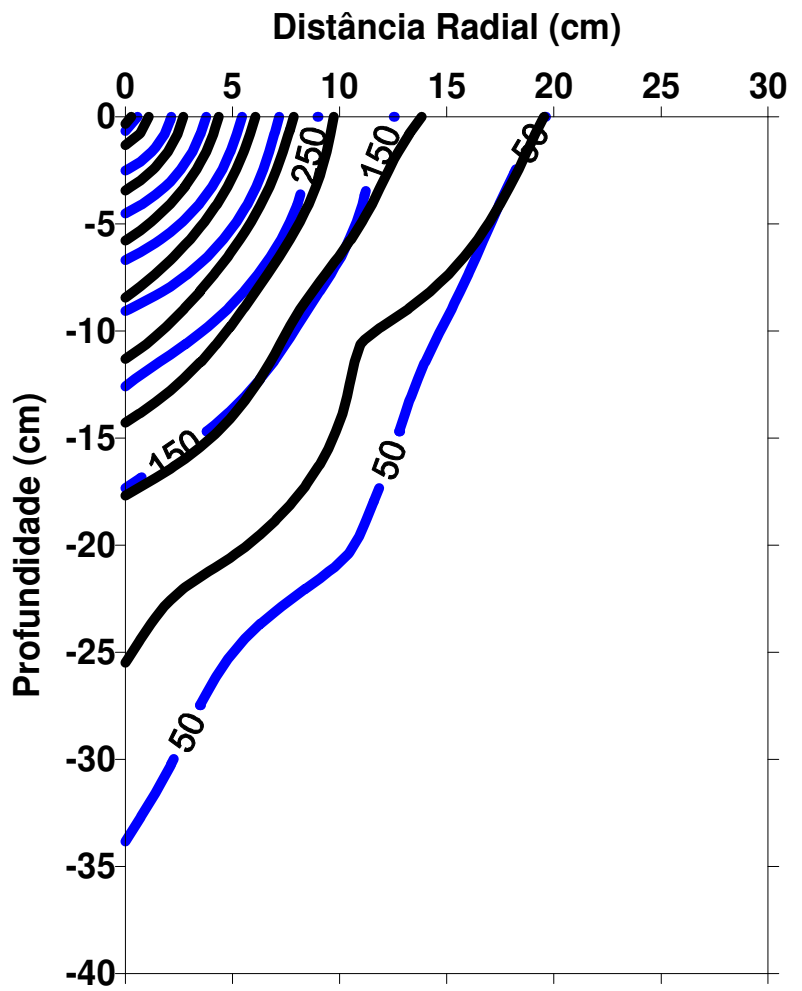

FIGURA 2. Concentração do potássio $\left(\mathrm{mg} \mathrm{L}^{-1}\right)$ no bulbo, simulada e observada, $24 \mathrm{~h}$ após a irrigação.

Em termos gerais, nos três tempos de redistribuição considerados, o conteúdo de potássio no solo simulado pelo modelo foi similar para as condições de experimentação e o deslocamento acompanhou parcialmente o deslocamento da água. O potássio apresentou taxas muito baixas de redistribuição e concentrou-se na superfície do solo e nas proximidades da fonte puntiforme, o que aparentemente demonstra que, além de ser retido nas camadas superiores ao ser adsorvido pela matriz do solo, o mesmo é transportado fundamentalmente pelo fluxo convectivo, junto com a água (denominado também de fluxo de massa), em forma proporcional à sua concentração (PREVEDELLO, 1996).

\section{CONCLUSÕES}

Houve bom ajuste dos valores da distribuição conjunta de água e potássio no bulbo quando se compararam os dados simulados pelo modelo com os dados obtidos experimentalmente. A 
distribuição do potássio ficou limitada às camadas superficiais do bulbo, sendo o deslocamento do cátion retardado pela adsorção da matriz do solo.

\section{REFERÊNCIAS}

BOTREL, T.A. Simulação da distribuição espacial da água em solo irrigado com gotejador. 1988. 80 f. Tese (Doutorado em Solos e Nutrição de Plantas) - Escola Superior de Agricultura "Luiz de Queiroz”, Universidade de São Paulo, Piracicaba, 1988.

BRANDT, A.; BRESLER, E.; DINER, N.; BEM-ASHER, J.; HELLER, J.; GOLDBERG, D. Infiltration from trickle source: I mathematical models. Soil Science Society of America Proceedings, Knoxville, v.35, n.5, p.675-82, 1971.

CLOTHIER, B.E. Solute travel times during trickle irrigation. Water Resources Research, Washington, v.20, n.12, p.1848-52, 1984.

CRUZ, R.L. Modelização do balanço hídrico de uma cultura irrigada por um sistema de irrigação localizada. 2000. 80 f. Tese (Livre-Docência em Irrigação e Drenagem) - Faculdade de Ciências Agronômicas, Universidade Estadual Paulista “Júlio de Mesquita Filho”, Botucatu, 2000.

EMPRESA BRASILEIRA DE PESQUISA AGROPECUÁRIA. Serviço Nacional de Levantamento e Conservação de Solos. Manual de métodos de análise do solo, 1997. 212 p.

GENUCHTEN, M.T. Van. A closed-form equation for predicting the hydraulic conductivity of unsaturated soils. Soil Science Society of America Journal, Madison, v.44, n.3, p.892-8, 1980.

KENGET, J.C.W.; SCOTT, T.W.; LUGO-LOPEZ, M. A fertilizer management with drip irrigation in a oxisol. Agronomy Journal, Madison, v.71, n.6, p.971-80, 1979.

MALAVOLTA, E. Potássio, magnésio e enxofre nos solos e culturas brasileiras. 2.ed. Piracicaba: Instituto Internacional de Pesquisa da Potassa, 1980. 91 p. (Boletim Técnico, 4)

MMOLAWA, K.; OR, D. Root zone solute dynamics under a drip irrigation: a review. Plant and Soil, Wageningen, v.22, n.2, p.163-90, 2000.

PREVEDELLO, C.L. Física do solo com problemas resolvidos. Curitiba: Sociedade Autônoma de Estudos Avançados em Física do Solo, 1996. 446 p.

RAIJ, B. Van; QUAGGIO, J.A. Métodos de análise de solos para fins de fertilidade. Campinas: Instituto Agronômico, 1983. 52 p. (Boletim Técnico, 81).

WARRICK, A.W. Time-dependent linearized infiltration: i) point source. Soil Science Society American Journal, Madison, v.38, n.3, p.383-6, 1974.

WARRICK, A.W. Point and line infiltration-calculations of the wetted soil surface. Soil Science Society American Journal, Madison, v.49, n.6, p.1581-3, 1985.

ZANINI, J.R. Distribuição de água e do íon K+ no solo, aplicados por fertirrigação em gotejamento. II - Teores de K+ no bulbo molhado. ITEM - Irrigação e Tecnologia Moderna, Brasília, v.46, n.1, p.24-38, 1991. 